\title{
La résistance aux antimicrobiens de Neisseria gonorrhoeae au Canada : 2009-2013
}

\author{
Martin $\mathrm{I}^{1, *}$, Sawatzky $\mathrm{P}^{1}$, Liu $\mathbf{G}^{1}$, Mulvey $\mathrm{MR}^{1}$ \\ ${ }^{1}$ Laboratoire national de microbiologie, Agence de la santé publique du Canada, Winnipeg (Manitoba) \\ *Auteure-ressource : Irene.Martin@phac-aspc.gc.ca
}

\section{Résumé}

Contexte : L'infection gonococcique est en expansion au Canada. Le traitement a été compliqué par le fait que la bactérie Neisseria gonorrhoeae a acquis une résistance à de nombreux antibiotiques, y compris la pénicilline, la tétracycline, l'érythromycine et la ciprofloxacine. L'émergence d'isolats présentant une sensibilité réduite aux céphalosporines de troisième génération et le signalement d'échecs de traitement au Canada et partout dans le monde sont préoccupants.

Objectif : Évaluer les niveaux de résistance de $N$. gonorrhoeae aux antibiotiques communs et observer les tendances de sa résistance ou de sa sensibilité réduite à la ciprofloxacine, aux céphalosporines de troisième génération et à l'azithromycine.

Méthodologie : On a comparé les données de surveillance en laboratoire d'isolats de $N$. gonorrhoeae présentées par des laboratoires provinciaux de microbiologie au Laboratoire national de microbiologie (LNM) de 2009 à 2013.

Résultats : Depuis 2009, on a observé une augmentation globale de bactéries $N$. gonorrhoeae résistantes aux antibiotiques. En 2013, 24,3\% des isolats étaient résistants à l'érythromycine, 18,9\% étaient résistants à la pénicilline, 33,0 \% étaient résistants à la tétracycline et $29,3 \%$ étaient résistants à la ciprofloxacine. Le pourcentage des isolats présentant une sensibilité réduite à la ceftriaxone $(\geq 0,125 \mathrm{mg} / \mathrm{L})$ ou à la céfixime ( $\geq 0,25 \mathrm{mg} / \mathrm{L}$ ) était de $3,9 \%$ en 2013. Ce nombre représente une baisse par rapport au pourcentage de 2012 qui était de $5,9 \%$ et à celui de 2011 qui était de 7,6 \%. La proportion d'isolats de $N$. gonorrhoeae résistants à l'azithromycine (CMI $\geq 2 \mathrm{mg} / \mathrm{L}$ ) a augmenté, passant de $0,4 \%$ en 2009 à 1,2 \% en 2013 .

Conclusion : La résistance à l'érythromycine, à la pénicilline, à la tétracycline et à la ciprofloxacine est courante. La sensibilité réduite à la ceftriaxone et à la céfixime est maintenant à une valeur de près de $4 \%$ et la résistance à l'azithromycine est émergente, mais elle demeure faible à $1,2 \%$. Ces résultats ont éclairé les recommandations en matière de traitement de l'infection gonococcique formulées dans les lignes directrices canadiennes sur les infections transmissibles sexuellement.

\section{Introduction}

$N$. gonorrhoeae, agent causal de l'infection gonococcique, est la deuxième infection bactérienne transmissible sexuellement la plus couramment déclarée au Canada et les taux de cas déclarés ont plus que doublé entre 1997 et 2012 (1).

Le traitement et le contrôle de l'infection gonococcique sont compliqués par la capacité de $N$. gonorrhoeae à évoluer rapidement et à développer une résistance à de nombreux antibiotiques utilisés pour la traiter $(2,3)$. L'émergence des isolats présentant une sensibilité réduite aux céphalosporines $(4,5,6,7)$ ainsi que le signalement d'échecs de traitement au Canada (8) et dans le monde entier soulèvent la possibilité que l'infection gonococcique devienne impossible à traiter à l'avenir. L'émergence de $N$. gonorrhoeae présentant une résistance élevée à l'azithromycine ( $\geq 256 \mathrm{mg} / \mathrm{L}$ ) a été signalée à l'échelle internationale (9) et des isolats présentant ce haut niveau de résistance à l'azithromycine ont maintenant été signalés au Canada.

Le nombre de cultures disponibles pour les tests de sensibilité aux antimicrobiens est à la baisse en raison du remplacement de l'utilisation de cultures par des tests d'amplification des acides nucléiques (TAAN) pour le diagnostic de l'infection gonococcique. Cette situation est préoccupante, car des cultures de $N$. gonorrhoeae sont 
nécessaires pour effectuer des tests de sensibilité aux antimicrobiens, et certaines provinces et certains territoires au Canada n'ont plus la capacité de maintenir la mise en culture de cet organisme. En fait, plus de $70 \%$ des infections gonococciques au Canada sont maintenant diagnostiquées au moyen de TAAN. Par conséquent, les données sur la sensibilité aux antimicrobiens dans ces provinces et territoires ne sont pas disponibles. Le Laboratoire national de microbiologie (LNM), en collaboration avec les laboratoires provinciaux, a effectué le suivi de la sensibilité aux antimicrobiens de N. gonorrhoeae depuis 1985.

L'objectif du présent relevé est de résumer les tendances en matière de résistance aux antimicrobiens des infections gonococciques au Canada entre 2009 et 2013. II est fondé sur le Rapport sommaire annuel de 2013 Surveillance nationale de la sensibilité aux antimicrobiens de Neisseria gonorrhoeae préparé par le LNM, Agence de la santé publique du Canada (ASPC) (10).

\section{Méthodologie}

\section{Collecte de données}

Les laboratoires provinciaux de santé publique ont soumis un total de 5518 isolats viables de N. gonorrhoeae au LNM aux fins de tests de sensibilité aux antimicrobiens dans le cadre du Programme national de surveillance passive de Neisseria gonorrhoeae entre 2009 et 2013 (2009, $N=913 ; 2010, N=1233 ; 2011, N=1158 ; 2012$, $N=1031 ; 2013, N=1183$ ). Les isolats de $N$. gonorrhoeae sont soumis au LNM si les laboratoires provinciaux décèlent une résistance à au moins un antibiotique ou s'ils n'effectuent aucun test de sensibilité aux antimicrobiens. La soumission d'isolats est volontaire et n'est pas uniforme dans l'ensemble du pays.

L'interprétation globale des résultats est difficile en raison des limites liées aux isolats disponibles pour les tests. Par conséquent, le nombre total d'isolats mis en culture dans toutes les provinces a été utilisé comme dénominateur pour calculer la proportion de résistance $(2009, N=3106 ; 2010, N=2$ 970; 2011, $N=3360$; 2012, $N=3$ 036; 2013, N = 3 195).

Les CMI ont été déterminées par dilution en gélose (11) et la résistance a été définie selon le Clinical and Laboratory Standards Institute (11), à l'exception de l'érythromycine (12) et de l'azithromycine (13). Les valeurs seuils de la sensibilité réduite à la ceftriaxone et à la céfixime étaient fondées sur les définitions de l'OMS (14). On a mis au point des abréviations communes pour les différents types de résistance (Tableau 1).

Tableau 1 : Abréviations et définitions pour les différents types de résistance antimicrobienne de Neisseria gonorrhoeae

\begin{tabular}{|c|c|c|}
\hline Abréviation & Expression au long & Définition \\
\hline NGPP & N. gonorrhoeae produisant une pénicillinase & $\begin{array}{l}\text { CMI Pén } \geq 2,0 \mathrm{mg} / \mathrm{L} \text {, positif pour } \beta \text {-lactamase, plasmide } \beta \text { - } \\
\text { lactamase (plasmide de } 3,05,3,2 \text { ou } 4,5 \mathrm{Md} \text { ) }\end{array}$ \\
\hline NGRT & $\begin{array}{l}\text { N. gonorrhoeae résistant à la tétracycline (médiation } \\
\text { plasmidique) }\end{array}$ & $\begin{array}{l}\text { CMI Tét } \geq 16,0 \mathrm{mg} / \mathrm{L} \text {, plasmide de } 25,2 \mathrm{Md} \text {, positif pour réaction } \\
\text { de polymérisation en chaîne de Tét } \mathrm{M}\end{array}$ \\
\hline NGRMC & N. gonorrhoeae résistant à médiation chromosomique & $\begin{array}{l}\text { CMI Pén } \geq 2,0 \mathrm{mg} / \mathrm{L} \text {, CMI Tét } \geq 2,0 \mathrm{mg} / \mathrm{L} \text { mais } \leq 8,0 \mathrm{mg} / \mathrm{L} \text { et } \mathrm{CMI} \\
\text { Éry } \geq 2,0 \mathrm{mg} / \mathrm{L}\end{array}$ \\
\hline NGRMC probable & $\begin{array}{l}\text { N. gonorrhoeae présentant une résistance à médiation } \\
\text { chromosomique probable }\end{array}$ & $\begin{array}{l}\text { Une des valeurs de CMI de Pén, Tét, Éry = } 1 \mathrm{mg} / \mathrm{L} \text {, les deux } \\
\text { autres } \geq 2,0 \mathrm{mg} / \mathrm{L}\end{array}$ \\
\hline RTét & $\begin{array}{l}\text { N. gonorrhoeae résistant à la tétracycline } \\
\text { (médiation chromosomique) }\end{array}$ & MIC Tét $\geq 2,0 \mathrm{mg} / \mathrm{L}$ mais $\leq 8,0 \mathrm{mg} / \mathrm{L}$ \\
\hline RCip & N. gonorrhoeae résistant à la ciprofloxacine & CMI Cip $\geq 1,0 \mathrm{mg} / \mathrm{L}$ \\
\hline RAz & N. gonorrhoeae résistant à l'azithromycine & $\mathrm{CMI} \mathrm{Az} \geq 2,0 \mathrm{mg} / \mathrm{L}$ \\
\hline RSpec & N. gonorrhoeae résistant à la spectinomycine & $\mathrm{R} \mathrm{Spec} \geq 128 \mathrm{mg} / \mathrm{L}$ \\
\hline SRCX & $\begin{array}{l}\text { N. gonorrhoeae ayant une sensibilité réduite à la } \\
\text { ceftriaxone }\end{array}$ & CMI Cx $\geq 0,125 \mathrm{mg} / \mathrm{L}$ \\
\hline SRCe & $\begin{array}{l}\begin{array}{l}\text { N. gonorrhoeae ayant une sensibilité réduite à la } \\
\text { céfixime }\end{array} \\
\end{array}$ & $\mathrm{CMI} \mathrm{Ce} \geq 0,25 \mathrm{mg} / \mathrm{L}$ \\
\hline
\end{tabular}




\section{Résultats}

La Figure 1 illustre les tendances de la sensibilité aux antimicrobiens des isolats de $N$. gonorrhoeae testés au Canada de 2009 à 2013.

Figure 1 : Tendances de la sensibilité aux antimicrobiens des isolats de Neisseria gonorrhoeae testés au Canada de 2009 à 2013

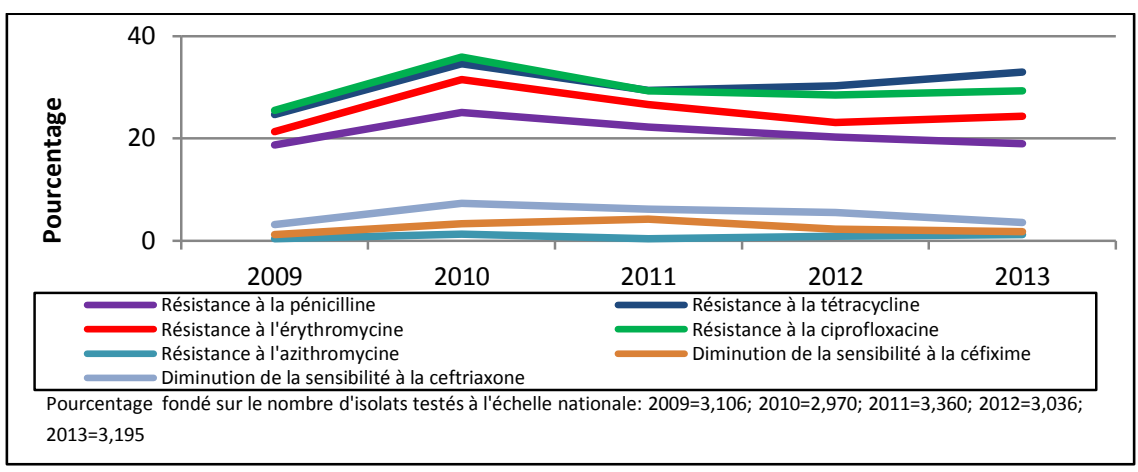

Des 3195 isolats de N. gonorrhoeae mis en culture dans les laboratoires de santé publique partout au Canada en 2013, 1183 isolats résistants présumés ont été soumis au LNM. De ce nombre, on a confirmé la résistance de 1153 isolats à au moins un antibiotique et 30 étaient sensibles, ce qui correspond à un taux de 36,1\% de tous les cas diagnostiqués par culture de $N$. gonorrhoeae résistant.

Les données sur l'âge et le sexe étaient disponibles pour 99,5\% des isolats testés par le LNM en 2013. De ce nombre, 83,1 \% étaient des hommes dont l'âge variait de 1 mois à 74 ans. Un total de 16,9 \% des isolats ont été prélevés chez des femmes âgées de 2 à 71 ans.

\section{Céphalosporines de troisième génération}

En 2013, selon les définitions de l'OMS, 1,8\% des isolats présentaient une sensibilité réduite à la céfixime et $3,5 \%$, une sensibilité réduite à la ceftriaxone. Ces taux sont plus élevés qu'en 2009 (1,2\% présentaient une sensibilité réduite à la céfixime et 3,1\%, une sensibilité réduite à la ceftriaxone), mais plus faibles qu'en 2011 (4,2\% présentaient une sensibilité réduite à la céfixime et à $6,2 \%$, une sensibilité réduite à la ceftriaxone). En 2013, 3,9\% des isolats ont été identifiés comme présentant une sensibilité réduite à la ceftriaxone ou à la céfixime, ce qui représente une diminution par rapport au taux de 5,9\% en 2012 et de 7,6 \% en 2011 .

\section{Azithromycine}

Le taux de N. gonorrhoeae résistant à l'azithromycine a augmenté, passant de 0,4\% en 2009 à 1,2 \% en 2013 . De 2009 à 2012, cinq isolats présentant une résistance élevée à l'azithromycine (CMI $\geq 256 \mathrm{mg} / \mathrm{L})$ ont été recensés au Canada. La CMI modale pour l'azithromycine est demeurée à 0,5 mg/L chaque année de 2009 à 2012. En 2013, la modale a diminué à $0,25 \mathrm{mg} / \mathrm{L}$.

En 2012, on a déterminé sept isolats présentant une sensibilité réduite combinée aux céphalosporines et une résistance à l'azithromycine $(0,2 \%)$. En 2013 , on a déterminé huit $(0,3 \%)$ de ces isolats. Il s'agit des premiers isolats à se manifester au Canada avec une sensibilité réduite aux céphalosporines et une résistance à l'azithromycine, menaçant donc la réussite des options de traitement à double modalité actuellement recommandées. 


\section{Autres antibiotiques}

Le pourcentage d'isolats résistants à la ciprofloxacine a augmenté, passant de $25,5 \%$ en 2009 à $29,3 \%$ en 2013. La résistance à la ciprofloxacine a augmenté, passant de 1,3\% en 2000 à un sommet de $36,0 \%$ en 2010. La CMI modale pour la ciprofloxacine est passée de façon radicale de 0,004 mg/L en 2004 à $16,0 \mathrm{mg} / \mathrm{L}$ en 2013 (données non indiquées).

En 2009, on a déterminé que $21,3 \%$ des isolats étaient résistants à l'érythromycine. Ce pourcentage a augmenté jusqu'à $31,5 \%$ en 2010 , puis a diminué à $24,3 \%$ en 2013. La résistance à la pénicilline a augmenté, passant de $18,7 \%$ en 2009 , à $25,1 \%$ en 2010 , puis elle a diminué à $18,9 \%$ en 2013 . La résistance à la tétracycline a augmenté, passant de $24,7 \%$ en 2009 à $34,6 \%$ en 2010, puis a diminué à $33,0 \%$ en 2013. Des 5518 isolats viables testés par le LNM de 2009 à 2013, aucun n'a présenté de résistance à la spectinomycine.

En 2013, 13,5\% des isolats ont été classés dans la catégorie de $N$. gonorrhoeae présentant une résistance à médiation chromosomique (NGRMC), une légère baisse par rapport au taux de 15,3\% observé en 2009. Les isolats de $N$. gonorrhoeae produisant une pénicillinase (NGPP) représentaient $4,3 \%$ en 2013 , une légère augmentation par rapport au taux de $2,5 \%$ en 2009. Le taux d'isolats de $N$. gonorrhoeae présentant une résistance à la tétracycline (NGRT) à médiation plasmidique a augmenté, passant de $3,2 \%$ en 2009 à $8,8 \%$ en 2013 (Figure 2).

Figure 2 : Tendances en matière de résistance antimicrobienne de Neisseria gonorrhoeae à médiation chromosomique et plasmidique au Canada de 2009 à 2013

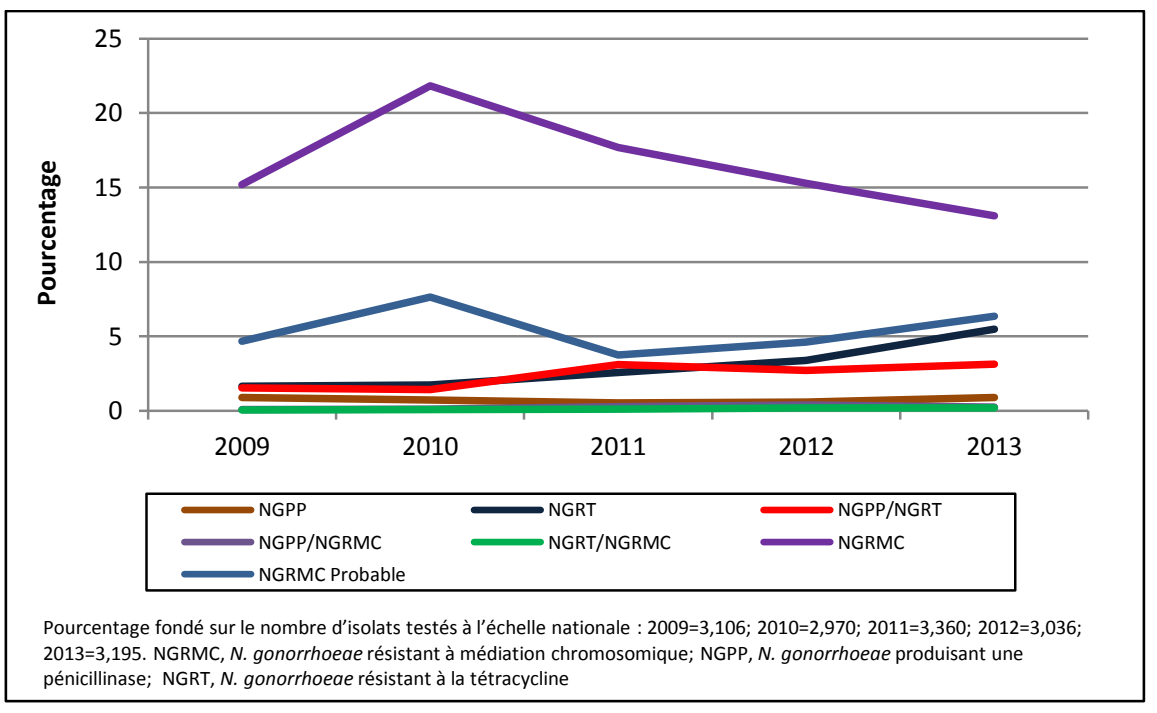

\section{Discussion}

L'évolution de la résistance de l'infection gonococcique aux antimicrobiens est complexe et la propagation d'isolats résistants est une menace reconnue pour la santé publique mondiale. La surveillance et le suivi de la sensibilité de $N$. gonorrhoeae aux antimicrobiens continuera à orienter les efforts visant à atténuer les répercussions de la résistance de l'infection gonococcique aux antimicrobiens et de guider les recommandations thérapeutiques.

Le signalement d'échecs de traitements à la céfixime et l'augmentation lente et progressive de la CMI observée de 2001 à 2010 , tant pour la céfixime (de $0,016 \mathrm{mg} / \mathrm{L}$ à $0,125 \mathrm{mg} / \mathrm{L})$ que pour la ceftriaxone $($ de $0,016 \mathrm{mg} / \mathrm{L}$ à $0,063 \mathrm{mg} / \mathrm{L}$ ), ont mené à des modifications du traitement de l'infection gonococcique. En 2011, les lignes directrices canadiennes sur les ITS ont mis à jour les recommandations sur l'utilisation d'un traitement combiné de l'infection gonococcique par $250 \mathrm{mg}$ de ceftriaxone par voie intramusculaire et $1 \mathrm{~g}$ d'azithromycine par voie 
orale comme traitement de première intention chez les hommes ayant des relations sexuelles avec d'autres hommes (HARSAH) et chez les personnes atteintes de pharyngite (15).

Depuis les modifications apportées en 2011 aux recommandations de traitement de l'infection gonococcique au Canada, on a observé une diminution de la proportion d'isolats présentant une CIM élevée aux céphalosporines. En $2011,7,6 \%$ des isolats présentaient une sensibilité réduite à la ceftriaxone ou à la céfixime, selon la définition de l'OMS. Cette proportion est passée à $5,9 \%$ en 2012, puis a diminué encore pour atteindre 3,9\% des isolats testés en 2013.

Heureusement, des taux décroissants de sensibilité réduite à la céfixime sont également observés ailleurs. Par exemple, les États-Unis ont signalé une diminution de la sensibilité réduite à la céfixime qui est passée de 3,9\% en 2010 à 2,9\% au cours de la première moitié de 2012 (16). Le Royaume-Uni a signalé une chute de la prévalence d'isolats présentant une diminution de la sensibilité à la céfixime qui est passée de 17,1 \% en 2010 à $10,8 \%$ en $2011(17)$.

L'amélioration de la surveillance pour inclure les liens entre les données épidémiologiques et les données de laboratoire permettra de contrer les limites du système actuel de surveillance passive en ce qui concerne la représentativité et l'interprétation des données. Ces améliorations du programme de surveillance gonococcique sont prévues dans le cadre du programme de système de surveillance accrue de la gonorrhée aux antimicrobiens (SARGA) qui sera lancé en 2014.

\section{Remerciements}

Le relevé dans son intégralité a été préparé par le Laboratoire national de microbiologie et le Centre de la lutte contre les maladies transmissibles et les infections de la Direction générale de la prévention et du contrôle des maladies infectieuses de l'Agence de la santé publique du Canada. Sa publication n'aurait pas été possible sans les données fournies par l'ensemble des provinces et territoires par l'intermédiaire du Réseau des laboratoires de santé publique du Canada (RLSPC). Nous leur sommes très reconnaissants de leur contribution continue au programme de surveillance nationale de Neisseria gonorrhoeae.

\section{Conflit d'intérêts}

Aucun

\section{Financement}

Ce travail a été appuyé par l'Agence de la santé publique du Canada.

\section{Références}

(1) Public Health Agency of Canada. Notifiable Diseases On-Line. 2014. http://dsol-smed.phac-aspc.gc.ca/dsolsmed/ndis/charts.php?c=pl. (disponible en français: http://dsol-smed.phac-aspc.gc.ca/dsolsmed/ndis/graphiques.php?c=pl.)

(2) Barry PM, Klausner JD. The use of cephalosporins for gonorrhoea: The impending problem of resistance. Expert Opin Pharmacother. 2009; 10:555-577.

(3) Tapsall J. Antibiotic resistance in Neisseria gonorrhoeae is diminishing available treatment options for gonorrhoea: Some possible remedies. Expert Rev Anti Infect Ther. 2006; 4:619-628.

(4) Golparian D, Hellmark B, Fredlund H, Unemo M. Emergence, spread and characteristics of Neisseria gonorrhoeae isolates with in vitro decreased susceptibility and resistance to extended-spectrum cephalosporins in Sweden. Sex Transm Infect. 2010; 86:454-460.

(5) Ison CA, Hussey J, Sankar KN, Evans J, Alexander S. Gonorrhoea treatment failures to cefixime and azithromycin in England, 2010. Euro Surveill. 2011; 16(14):pii=19833.

(6) Pandori M, Barry PM, Wu A, Ren A, Whittington WLH, Liska S, Klausner JD. Mosaic penicillin-binding protein 2 in Neisseria gonorrhoeae isolates collected in 2008 in San Francisco, California. Antimicrob Agents Chemother. 2009; 53:4032-4034. 
(7) World Health Organization (WHO). 2011. Emergence of multi-drug resistant Neisseria gonorrhoeae: Threat of global rise in untreatable sexually transmitted infections. http://whqlibdoc.who.int/hq/2011/WHO_RHR_11.14_eng.pdf

(8) Allen VG, Mitterni L, Seah C, Rebbapragada A, Martin IE, Lee C, Siebert H, Towns L, Melano RG, Lowe DE. Neisseria gonorrhoeae treatment failure and susceptibility to cefixime in Toronto, Canada. JAMA. 2013; 309:163-170.

(9) Chisholm SA, Neal TJ, Alawattegama AB, Birley HDL, Howe RA, Ison CA. Emergence of high-level azithromycin resistance in Neisseria gonorrhoeae in England and Wales. J Antimicrob Chemother. 2009; 64:353-358.

(10) Public Health Agency of Canada. National Microbiology Laboratory. 2014. National surveillance of antimicrobial susceptibilities of Neisseria gonorrhoeae annual summary 2013.

(11) Clinical and Laboratory Standards Institute. Performance standards for antimicrobial susceptibility testing: Twenty-third informational supplement M100-S24 Vol. 34. Wayne, PA: Clinical and Laboratory Standards Institute; 2014.

(12) Ehret JM, Nims LJ, Judson FN. A clinical isolate of Neisseria gonorrhoeae with in vitro resistance to erythromycin and decreased susceptibility to azithromycin. Sex Transm Dis. 1996; 23:270-272.

(13) Centers for Disease Control and Prevention. Sexually transmitted disease surveillance 2007 Supplement, gonococcal isolate surveillance project (GISP) Annual report 2007. Atlanta, GA: U.S. Department of Health and Human Services, Centers for Disease Control and Prevention, March 2009. http//www.cdc.gov/std/GISP2007/

(14) World Health Organization (WHO). 2012. Global action plan to control the spread and impact of antimicrobial resistance in Neisseria gonorrhoeae. http://www.who.int/reproductivehealth/publications/rtis/9789241503501/en/ .

(15) Public Health Agency of Canada 2011.Important Notice- Public Health Information Update on the Treatment for Gonococcal Infection - Public Health Agency of Canada Issued Important Notice on gonococcal infection. (disponible en français : http://www.phac-aspc.gc.ca/std-mts/sti-its/alert/2011/alert-gono-fra.php)

(16) Kirkcaldy RD, Kidd S, Weinstock HS, Papp JR, Bolan GA. Trends in antimicrobial resistance in Neisseria gonorrhoeae in the USA: the Gonococcal Isolate Surveillance Project (GISP), January 2006-June 2012. Sex Transm Infect. 2013 Dec; 89 Suppl 4:iv5-10.

(17) Ison CA, Town K, Obi C, Chisholm S, Hughes G, Livermore DM, Lowndes CM, GRASP Collaborative Group. Decreased susceptibility to cephalosporins among gonococci: Data from the Gonococcal Resistance to Antimicrobials Surveillance Programme (GRASP) in England and Wales, 2007-2011. Lancet Infect Dis. 2013; 13:762-8. 